\title{
La delgada línea entre derechos humanos, comunicación y gênero
}

\author{
Maricela López-Ornelas ${ }^{1}$ \\ Cecilia Osuna Lever ${ }^{2}$ \\ Karla María Díaz López 3
}

\begin{abstract}
Resumen: El trabajo de corte teórico, aborda brevemente algunas aproximaciones que delinean la estrecha y polisémica relación entre derechos humanos, comunicación y género. Cabe reconocer, que dichos conceptos - entretejidos en las ciencias sociales-, han sido explicados y reinterpretados en diversos ámbitos a través de las propias trasformaciones socioculturales existentes, por lo que el documento no ahondará necesariamente en definiciones, pues habría que situar cada significado en las diversas áreas en las que estas nociones continúan inmersas. Bajo esta reflexión, el objetivo del artículo provee tres vertientes, identificar la delgada relación entre derechos humanos, comunicación y género, dando énfasis al "derecho a comunicar" como un derecho inherente al ser humano, en un segundo aspecto, se aborda la visible postura sexista que amplía la brecha de desigualdad entre hombres y mujeres en el ámbito de la comunicación como profesión, y tercero, no menos relevante, se plantea que en países como México, coexiste una violación continua sobre el "derecho de comunicar". Entre los resultados se evidencia que la perspectiva de género, es una construcción cultural que incide directamente en el composición de los derechos humanos, misma que confirma que la comunicación es considerada clave en el desarrollo de la configuración de las prácticas sociales sobre los derechos humanos más elementales; además, se identifica en la literatura, que pese a diversos esfuerzos, las mujeres siguen deficientemente representadas en el contenido mediático; finalmente, México es señalado como el sexto país con el mayor número de violaciones a los derechos humanos de los comunicadores, independientemente a su género.
\end{abstract}

Palabras-Clave: Comunicación. Derechos Humanos. Derecho a Informar. Género. Libertad De Expresión.

\begin{abstract}
The theoretical work, briefly addresses some approaches that delineate the narrow and polysemous relationship between human rights, communication and gender. It should be recognized that these concepts - interwoven in the social sciences - have been explained and reinterpreted in various fields through the existing sociocultural transformations, so the document will not necessarily delve into definitions, since each meaning should be placed in the diverse areas in which these notions continue immersed. Under this reflection, the objective of the article provides three aspects, identifying the thin relationship between human rights, communication and gender, emphasizing the "right to communicate" as an inherent right to the human being; in a second aspect, the visible position is addressed sexist that widens the inequality gap between men and women in the field of communication as a profession, and third, no less relevant, states that in countries like Mexico, a continuous violation of the "right to communicate" coexists. The results show that the gender perspective is a cultural construction that directly affects the composition of human rights, which confirms that communication is considered key in the development of the configuration of social practices on human rights more elementary In addition, it is identified in the literature, that despite various efforts, women are still poorly represented in the media content; finally, Mexico is indicated as the sixth country with the highest number of violations of human rights of communicators, regardless of their gender.
\end{abstract}

Keywords: Communication; Human Rights; Gender; Right to Inform; Freedom of Expression.

1 Doutora em Comunicação pela Universidad de La Laguna, Espanha. E-mail: ornelas@uabc.edu.mx

2 Doutora em Ciências Educativas pelo Instituto de Investigación y Desarrollo Educativo de la Universidad Autónoma de Baja California, México. E-mail: cecilia.osuna@cetys.mx

3 Doutora Ciências Educativas pelo Instituto de Investigación y Desarrollo Educativo IIDE-UABC.Email: karla.diaz@cetys.mx 


\section{Introducción}

\section{Un primer acercamiento a la delgada línea entre derechos humanos, comunicación y género}

Aproximarse al tema de los derechos humanos en cualquier investigación, además de prometer un análisis complejo, también resulta visiblemente comprometedor, pues "confronta diferentes valores que tienen relación con principios que son muy subjetivos" (FRAU-MEIGS, 2012, p. 104), aun así, y con este matiz interpretativo, el tópico se "ha convertido en clave del desarrollo político, social y económico en todo el mundo (SREBERNY, 2012, p. 13).

Algo similar sucede al pretender delimitar las líneas entre comunicación y género, pues la polémica relación con los derechos humanos se encuentra intrínsecamente vinculada a la comunicación (WASKO, 2012), y es ahí, donde percibimos que la intervención social derivada de la comunicación moderna, es uno de los procesos más concluyentes en la fecundación de las ideologías de género (CABALLERO, 2017), en este sentido, el campo académico y profesional de estudio y práctica - de la comunicación-, establece una correspondencia inseparable a los derechos humanos, como resultado, no se puede desatender el histórico debate ideológico-constitucional sobre el “derecho a comunicar” (SREBERNY, 2012).

Bajo estas premisas, el objetivo de este artículo provee tres escenarios de análisis: intenta encuadrar la cambiante relación entre derechos humanos, comunicación y género, y cómo la significación de cada concepto permea en el campo del otro. De igual forma, espera exponer como un derecho inseparable al ser humano -el derecho a comunicar-, está provisto de matices sexistas que ensanchan la brecha de desigualdad de oportunidades entre hombres y mujeres - las cuales van más allá de los ámbitos laborales-. En la misma línea, no menos relevante, aborda el hecho de que independientemente del género, en países como México - entre otros-, existe una constante violación al "derecho de comunicar", que se censura a quienes lo ejercen de la forma más atroz e inhumanamente pensada, el homicidio. 


\section{La Organización de las Naciones Unidas (ONU), el mejor aliado de los Derechos Humanos}

Uno de los esfuerzos más sólidos y contundentes - a nivel internacionalen el tema de los derechos humanos, está representado en la Declaración Universal de Derechos Humanos (DUDH) -disponible en más de 360 idiomas-, y constituida por la Asamblea General de la Organización de las Naciones Unidas (ONU) desde diciembre de 1948, fecha en la que se estableció el primer instrumento normativo universal destinado a proclamar la igualdad, la dignidad y los derechos de todos los miembros de la familia humana (BOAFO, 2012), y que hasta hoy en día, a casi siete décadas de su instauración, es valorado como el documento en el que por primera vez se expresaron claramente los derechos y las libertades a los que todo ser humano de manera inalienable y en condiciones de igualdad debe tener (KI-MOO, 2015).

En consecuencia, aquellos artículos que promueven esta declaración, son de extrema importancia para alcanzar una convivencia de paz universal, por lo que su análisis podría enriquecer cualquier área de investigación. No obstante, para este trabajo - y con la idea de centrar el presente trabajo-, se retoma el Artículo 19 de la DUDH, el cual señala que:

\footnotetext{
"Todo individuo tiene derecho a la libertad de opinión y expresión; este derecho incluye el de no ser molestado a causa de sus opiniones, el de investigar y recibir informaciones y opiniones, el de difundirlas sin limitación de fronteras por cualquier medio de expresión" (DUDH, ART. 19).
}

Al vincular los derechos humanos con la libertad de opinión y expresión, se podría considerar que existe una diversidad de organismos que proveen normativas que regulan este derecho, sin embargo, la literatura evidencia que es un área que requiere todavía de un alto compromiso por parte de gobiernos y ciudadanos.

Con base a lo anterior, BOAFO (2012, pp. 39 y 41) expresa que la Organización de las Naciones Unidas para la Educación, la Ciencia y la Cultura (UNESCO por sus siglas en inglés), es la única agencia de la ONU con un mandato normativo en Comunicación e Información, que fomenta "el libre intercambio de ideas y conocimientos" y "el libre flujo de ideas a través de la palabra y la imagen”. 
Bajo estos planteamientos iniciales, la comunicación se ha mantenido vigente en el tema de los derechos humanos con la promulgación DUDH, y ha sido vector de diversos estudios y trabajos académicos, donde uno de sus ejes manifiesta que:

“...si los individuos no tienen derecho de buscar, recibir y difundir sus ideas y opiniones libremente, pudieran no beneficiarse de otros derechos humanos" (BOAFO, 2012, p. 41).

Es decir, el derecho que garantiza la libertad de expresión está fundamentalmente definido, como la parte medular de todos los demás derechos humanos, así como de las libertades democráticas, en donde la comunicación es la parte vertebral.

En el capítulo Comunicación y Derechos Humanos: el papel de la UNESCO, BOAFO (2012, p. 42) señala que en 1976, la Organización de las Naciones Unidas para la Educación, la Ciencia y la Cultura dio a conocer su postura ante la participación activa del proceso de la comunicación y los derechos humanos:

\begin{abstract}
"La cambiante situación mundial está produciendo profundas variaciones en la comunicación humana. Estos cambios están abriendo a su vez nuevas y más amplias perspectivas. Es evidente que hay una estrecha relación entre las necesidades humanas de la comunicación, el desarrollo de los recursos de comunicación y los derechos humanos. Esta relación puede enunciarse como sigue: la humanidad tiene derecho a los recursos de comunicación requeridos para satisfacer las necesidades de la comunicación humana. El derecho a la comunicación se basa en la necesidad de comunicación de todo ser humano" (UNESCO, 1976, p. 8).
\end{abstract}

Cuatro década después - aún prevalece el valor de la comunicación, no solo como derecho a ejercerla, sino como proceso comunicativo imprescindible en el ejercicio de los derechos humanos-, donde los embates del nuevo siglo los enfrentan a dos nuevas consideraciones: la globalización y el multiculturalismo (TALAVERA, 2011).

En estas consideraciones, la comunicación establece su propio proceso y ritmo, por tanto, se interpreta y re-interpreta, sumándose a los derechos humanos bajo una perspectiva socio-cultural y representativa que genera la cultura de la reflexión sobre las costumbres y tradiciones de todos los seres humanos. 
Por lo que la globalización ha transmitido un amplio conocimiento y una subjetiva conciencia sobre la diversidad cultural, mientras que la multiculturalidad ha sacudido nuestro modo de concebir e interpretar los derechos humanos. De manera que el multiculturalismo, es la puerta que ofrece una perspectiva al tema del género y a los derechos humanos, en tanto que la globalización provee el acceso a ese multiculturalismo.

\title{
Una aproximación multidisciplinar
}

\begin{abstract}
"Sólo la concepción de una lógica de la comunicación asentada en un a priori universal permite el reconocimiento de las diferencias de género, de modo que éstas redunden efectivamente en unas relaciones de género simétricas y, en consecuencia, en la autorrealización de varones y mujeres como sujetos responsables de su propio destino y como portadores de unos derechos inalienables, inherentes a la propia condición humana de todas las persona" (PHILIPP, 2010, p. 152).
\end{abstract}

De acuerdo a FAUR (2003, citado en BRUNO y GUERRINI, 2006, p. 42), erróneamente se supone que género constituye una forma más académica de decir mujer, y aunque la literatura toma postura al hablar de género refiriéndose particularmente al rol de la mujer en los medios de comunicación - para organismos como la Unesco, género involucra a hombres y mujeres-.

De manera que los estudios de género y derechos humanos, se han convertido en una línea de investigación a nivel internacional que intenta dar cuenta de los derechos - esencialmente-, por considerar que son "quienes de forma más flagrante y sistemática se ven constantemente privadas de sus derechos de poder desarrollarse libremente como personas en todos los rincones de nuestro mundo" (PHILIPP, 2010, pp. 136).

Con este punto de vista, dicha área de estudio ha cobrado impulso y fuerza en diferentes instituciones de educación superior, organismos oficiales y organizaciones no gubernamental - conocidas universalmente como ONG-, no solo como temática en los cuerpos académicos, congresos, foros y seminarios, incluso, existe una gran oferta de posgrados y programas de especialización con alto nivel que promueven la importancia de lo que originalmente surgió como movimientos a favor 
de la igualdad de género - pesando en las mujeres-. Sin embargo, aún con todos estos esfuerzos, pareciera que "no hay un solo tema en el que las mujeres superen en número a los hombres como hacedores de noticias” (GALLAGHER 2012, p. 89).

\section{El rol de los medios de comunicación en la construcción del concepto de género y derechos humanos}

Uno de los instrumentos globales que caracteriza el siglo XXI, y cuya función es representar, resignificar y afirmar los perfiles humanos, es sin duda el de los medios de comunicación de masas, pues no existe una mayor globalización que los medios (LOVERA, 2007).

De esta fusión surge el concepto de "enfoque de género en la comunicación”, ya que según BURCH (2007), la incursión de las mujeres en las diversas esferas de la actividad humana, representa uno de los cambios sociales más contundentes del fin de este siglo, particularmente en el ámbito de la comunicación. Por tanto, el mismo autor expresa que esto no sólo se ha convertido en un espacio de realización profesional, sino que dada la influencia que ejercen en la opinión pública, el enfoque de género en la comunicación ha sido identificada como un área estratégica para cambiar las relaciones de desigualdad entre los géneros, o en su defecto, para reforzar y perpetuarlas.

\footnotetext{
"Lograr reducir o erradicar los estereotipos de género en los medios exige una estrategia desde múltiples miradas, apoyado por un compromiso hacia la formación ciudadana que contemple y respete las diferencias de género, otorgando a la mujer el papel que le corresponde (GARCÍA-RUIZ, AGUADED y RODRÍGUEZ, 2015, p. 581).
}

De manera que la perspectiva de género, es una construcción cultural que incumbe directamente en el ejercicio de los derechos humanos. Por lo cual, la comunicación para el desarrollo la reconoce como una de las claves de lectura e intervención en las prácticas sociales (BRUNO y GUERRINI, 2006).

En los últimos años, numerosas organizaciones a nivel internacional se han adjudicado el reto de instaurar la introducción de una perspectiva de género en la comunicación, entendida como uno de los componentes fundamentales de su 
democratización. Es precisamente este, uno de los proyectos centrales que promueven la vigencia del derecho de las mujeres a la comunicación y la información como una condición fundamental para su plena participación ciudadana (BURCH, 2007).

En base a lo anterior, GALLAGHER (2012) expone que la censura de género, obscurece las verdaderas condiciones de vida de las mujeres. Al respecto, la autora plantea que si recordamos esta vertiente en nuestras investigaciones, arrojaremos más luz sobre los obstáculos de género específicos que deben ser diluidos con la finalidad de que las mujeres ejerzan sus derechos humanos y de comunicación (p. 93). En decir, conseguir aminorar o eliminar los estereotipos de género en los medios demanda estrategia multidisciplinarias, a favor de la formación ciudadana que reflexione y respete las diferencias de género, otorgando a la mujer el papel que le corresponde.

\section{Conferencias mundiales sobre la mujer. Un compromiso internacional a favor de la igualdad}

La Organización de las Naciones Unidas, ha sido punta de lanza sobre el tema de los derechos de la mujer, y sus principales acciones, correspondieron a las organización de cuatro conferencias mundiales sobre la mujer, realizadas en en 1975 en Ciudad de México, en 1980 en Copenhague, en 1985 Nairobi y la última registrada, fue en 1995 en Beijín.

La Cuarta Conferencia Mundial sobre la Mujer, celebrada en la República Popular China, emitió una significativa pronunciación para la agenda mundial sobre igualdad de género. La Declaración y Plataforma de Acción de Beijing, adoptada de forma unánime por 189 países, organizó un programa a favor del empoderamiento de la mujer y donde se elaboró un documento clave de política mundial sobre igualdad de género.

Estás cuatro conferencias mundiales sobre las mujeres, han respaldado y unido a la comunidad internacional con objetivos comunes: la estrategia de acción para el avance y la igualdad de las mujeres en todas partes y en todas las campos de la vida pública y privada (PHILIPP, 2010). 


\title{
En busca de la igualdad de género en los medios de comunicación. Una aproximación a través de la Unesco
}

\begin{abstract}
"El desarrollo sostenible, los derechos humanos y la paz solo se pueden lograr si mujeres y hombres gozan de amplias e iguales oportunidades para vivir en libertad y con dignidad. La igualdad existe cuando mujeres y hombres tienen igualdad de acceso a la educación de calidad, a los recursos y al trabajo productivo en todas las esferas y cuando tienen la posibilidad de compartir el poder y el conocimiento sobre esa base. La igualdad de género se debe entender como una necesidad práctica y como un requisito ético. Irina Bokova, Directora General" (UNESCO, 2014, p. 3).
\end{abstract}

La Unesco, a través del sector de Comunicación e Información, inició una tarea colosal, al establecer a nivel internacional una extensa gama de "iniciativas específicas de género que considera todos los medios y todas sus acciones". Su objetivo general, es luchar a favor de la "igualdad entre mujeres y hombres que trabajan en los medios de comunicación, así como la equidad en la información sobre mujeres y hombres", ya que tienen la misma importancia (UNESCO, 2014, p. 9).

En 1995, la ONU puso énfasis en el protagonismo clave de los medios de comunicación para promover la igualdad de género en todas las esferas; su objetivo rector, fue evitar "estereotipos sobre la mujer y desigualdad de acceso y participación de la mujer en todos los sistemas de comunicación, especialmente en los medios de difusión" (UNESCO, 2014, p. 9).

Para 2010, la Asamblea General de la ONU fundó la organización ONU Mujeres [1]. En el mismo rubro, la Unesco organizó el Plan de Acción prioritario sobre igualdad de género 2008-2013 con dos acciones clave:

1) creación de programas específicos de género $y$

2) transversalización de género en acciones de todas las esferas de competencia de la Unesco (ONU Mujeres, 2016).

De esta forma, la Organización de las Naciones Unidas para la Educación, la Ciencia y la Cultura, junto con la Federación Internacional de Periodistas y otros socios, produjeron Indicadores de Género para Medios de Comunicación (GSIM). Dicho proceso, se logró en dos años, iniciando a principios de 2010 con un debate mundial sobre la plataforma de defensa de la política y con el lema: "Las mujeres hacen las noticias". La finalidad de esta propuesta, ha sido encabezar un intercambio 
mundial sobre la importancia y la necesidad de crear indicadores de sensibilidad al género para las organizaciones de medios de comunicación (UNESCO, 2014).

A este esfuerzo, le siguió el Debate Temático concretado por el Programa Internacional para el Desarrollo de la Comunicación (PIDC), [2] donde el tema base de análisis fue "Género y medios de comunicación: alcanzar el equilibrio justo", donde se abordaron "un conjunto de indicadores para evaluar la igualdad de género y el empoderamiento de la mujer en los medios de comunicación, con el objetivo de que las organizaciones mediáticas examinaran sus propias políticas y prácticas internas a favor de la equidad" (UNESCO, 2016, s/p).

Esta iniciativa fue tan productiva, qué el PIDC le dio la bienvenida, lo que demostró la importancia del tema para países miembros de la Unesco. Cabe resaltar, que "el PIDC es el único foro multilateral del sistema de las Naciones Unidas que moviliza a la comunidad internacional para debatir sobre el desarrollo de los medios de comunicación y promoverlo en los países en desarrollo" (UNESCO, 2016, s/p).

El proyecto final, titulado Indicadores de Género para Medios de Comunicación. Marco de indicadores para evaluar la sensibilidad en materia de género en las operaciones y contenidos mediáticos, [3] comprende un documento de 207 páginas, publicado en 2014, cuya estructura contiene dos grandes categorías, de donde se desprende otras subcategorías:

A. Acciones que fomentan la igualdad de género dentro de las organizaciones de medios de comunicación.

1.1. Balance de género en el nivel de toma de decisiones.

1.2. Igualdad de género en el trabajo y condiciones de trabajo.

1.3. Igualdad de género en uniones, asociaciones, clubes y organizaciones de periodistas, otros profesionales y organismos autorreguladores de medios de comunicación.

1.4. Las organizaciones de medios de comunicación promueven códigos de ética y políticas editoriales a favor de la igualdad de género en el contenido mediático.

1.5. Balance de género en educación y capacitación. 
B. Representación del género en el contenido mediático.

2.1 Representación del género en noticias y actualidades

2.2 Representación del género en publicidad

No existen registros en la literatura sobre el éxito de este proyecto, no obstante, se debe reconocer que la propuesta es un logro por sí misma, máxime si convocaron a especialistas representantes de las organizaciones nacionales, regionales e internacionales en el campo de los medios de comunicación y género, de todas las regiones del mundo.

Actualmente, la Unesco cuenta con 195 estados miembros y 10 miembros asociados, organizados en cinco grupos regionales África, Estados Árabes, Asia y el Pacífico, Europa y América del Norte, América Latina y el Caribe. Lo que nos permite visualizar, que los esfuerzos que realiza este organismo a favor de los derechos humanos en materia de comunicación y género, esperan tener -mínimamente-, un impacto positivo con sus estados miembros. En este sentido, el documento titulado Indicadores de Género para Medios de Comunicación. Marco de indicadores para evaluar la sensibilidad en materia de género en las operaciones y contenidos mediáticos expone el valor que ha adquirido la comunicación y el género, en materia de derechos humanos, lo mismo sucede con el Model curricula for journalism education: a compendium of new syllabi, publicado por la Unesco en tres idiomas: inglés, chino y mongol, que si bien no se enfoca a cuestiones de género, es un excelente esfuerzo por para instituir estándares basados en buenas prácticas periodísticas a nivel internacional, por tanto, tiene por convicción, que los estándares periodísticos profesionales, esenciales para un sistema de medios, puedan fomentar la democracia, el diálogo y el desarrollo (UNESCO, 2016).

\section{Libertad de expresión y derechos humanos, más allá de las cuestiones de género}

Sin dejar de lado el tema cultural, los derechos humanos y las perspectivas de género, están intrínsecamente articulados con las dificultades para armonizar el 
derecho humano a la igualdad sobre "el derecho humano a la comunicación", lo que significa, asegurar que el potencial de mujeres y hombres - niñas y niños- sea liberado completamente y que el valor de cada persona sea reconocido sin prejuicios (UNESCO, 2014, p.9).

Es decir, uno de los aspectos clave referidos a la interacción entre la libertad de expresión, la igualdad y el derecho a la información, está puntualmente mencionado en el principio de no discriminación. Un sustento significativo lo otorga la Corte Interamericana de Derechos Humanos, quien estableció que "la libertad de expresión es sustancial para una sociedad democrática, ya que una sociedad que no está debidamente informada, se considera que no es una sociedad libre". Sin embargo, la identificación y el impulso de estos principios se han enfrentado a un ambiente complejo, incluso para los activistas de los derechos humanos y para la comunidad internacional, y sabemos que México no es la excepción (CALLAMARD, 2012, p. 52 y 53).

\section{México Una realidad que duele en materia de derechos humanos y que va más allá del género, pero sí del derecho a comunicar}

Los temas relacionados con la igualdad y los derechos humanos en México, recaen desenfrenadamente en una diversidad de ejemplos que traspasan lo inimaginable, y que históricamente siguen golpeando a los grupos más vulnerables de la sociedad, y si bien el objetivo de este trabajo desatiende el debate, se consideró inminente expresar la dolorosa diversidad en las que son trasgredidos los derechos humanos en nuestro país, algunos de los casos más conocidos, son los feminicidios, la trata de personas, los desparecidos - recordemos a los 43 normalistas-, el maltrato de nuestras mujeres indígenas, entre otros tantos, y sin dejar de lado esta apesadumbrada realidad, lo que nos acoge en este momento, es la incesante lista de periodistas asesinados en el ámbito laboral al ejercer su derecho a comunicar.

Este último tema, relacionado con el derecho promulgado en la Declaración Universal de Derechos Humanos, golpea con tal fuerza a México, que hace imperioso reconocer que nuestros colegas que trabajan en los medios de comunicación, se juegan la vida cotidianamente al profesionalizar un derecho universal, informar. 
Un ejemplo contundente se refleja en las cifras -números que tienen nombre, apellidos y familia-, y que indican que México en 2016, ocupó el séptimo lugar a nivel mundial -de 12 países-, y el primero en Latinoamérica, donde los periodistas son asesinados y los homicidas o autores intelectuales no han sido capturados o procesados legalmente. Para el 2017, retrocede desfavorablemente, ejerciendo el sexto puesto de acuerdo al Índice Global de Impunidad verificado por el Comité para la Protección de los Periodistas [4] (CPJ por sus siglas en inglés 2017).

El que existan otros 12 países [5] donde por lo menos cinco periodistas han sido asesinados sin haber condenado a nadie, no disimula el contexto que vivimos en México, [6] al contrario, mantiene un foco encendido sobre uno de los derechos humanos más básicos e importantes para los seres humanos, el comunicar.

\footnotetext{
"Frente a los numerosos desafíos del porvenir, la educación constituye un instrumento indispensable para que la humanidad pueda progresar hacia los ideales de paz, libertad y justicia social" (DELORS, AMAGI, CARNEIRO, CHUNG, GEREMEK, GORHAM, KORNHAUSER et al., 1997, p. 7).
}

Conscientes de la problemática, y en el entendido que "educar" es uno de los primeros pasos, las Naciones Unidas y su Organización para la Educación, la Ciencia y la Cultura, trabajaron en un programa de capacitación teórica y práctica para periodistas, con el objetivo de impulsar códigos de ética del periodismo, códigos voluntarios para los mismos gremios, no impuestos por ningún estado ni por una norma, con el objetivo de impulsar el respeto a las diferencias culturales y religiosas (LEWY 2012, p. 57). Dicho documento, se publicó en 2013, con el título, Unesco (2013) Model Curricula for Journalism Education. A Compendium of New Syllabi. [7].

El compendio de planes de estudio, aparece, por un lado, después de la más importante crisis económica global desde 1929 y, por otro, en plena explosión del cambio tecnológico. Ambos factores han cuestionado, desde la base educativa, los modelos industriales que soportan la mayoría de los medios de comunicación y, además, la formación que se imparte en las aulas para los futuros profesionales (SENDÍN, 2015, p.15). 
A nuevos tiempos, nuevos procesos, explica FRAU-MEIGS, en su capítulo "Educación en medios y derechos humanos", quien considera que el proceso generativo de los derechos humanos es más heurístico, más sistémico, más adaptado a las situaciones, con un fin abierto, "donde el Estado y el individuo tienen que estar implicados en los mecanismos para la resolución” (2012, p. 106).

\section{Reflexiones finales}

Como se comentó al inicio de este trabajo, el tema de derechos humanos es complicado por sí solo, e incluir al análisis la vigente necesidad de estudiar la comunicación en ese contexto - como proceso fundamental-, y a la vez considerar las perspectivas de género, hacen que el contenido, además de complejo, sea polémico e inagotable, y con seguridad para algunos lectores, no abordado con la seriedad y rigidez que las tres vertientes - derechos humanos, comunicación y género- lo requieren, incluso, podemos todavía atrevernos a decir, que tampoco fue planteado el orden correspondiente, y es que por sí solo, uno de estos temas es ilimitado, y su combinación lo hace más complejo. Respecto a esta ambigüedad, la UNESCO (2014, p. 15) pronuncia que "la multiplicación de los medios de comunicación, así como la estampida de las nuevas tecnologías y el surgimiento de los medios sociales en diversas partes del mundo, han generado la inclusión de múltiples fuentes de acceso a la información y a los conocimientos relativos al género y a los derechos humanos”. En este sentido, los medios tecnológicos, extienden el ámbito de la expresión y la comunicación a otros espacios antes privativos para la mayoría de los individuos (BUSTAMANTE, 2001)

De manera que es un hecho que nuestra desarrollada capacidad de expresarnos está complejizando nuestra libertad de expresión, pues los conceptos tradicionales no exteriorizan, ni el espíritu ni el camino para subsistir a los nuevos códigos y a los nuevos modos del comunicar (PASQUALI, 2012, p.120). A esta situación, se une la confusión de la pluralidad social, misma que simboliza una multiplicidad de características intrincadas en los seres humanos, como son el lenguaje, la cultura, la religión, la raza, la etnicidad y el género. Y para lograr que los 
medios de comunicación proyecten lo más real posible nuestras sociedades y promuevan una cobertura completa y diversa al momento de proyectar su visión del mundo, se vuelve inminente que las noticias -en particular- erradiquen los estereotipos y la masculinización, pues todo periodista puede desempeñar la labor de formar la opinión acerca de la igualdad de género a través de los medios de comunicación (UNESCO, 2014, p. 15).

\begin{abstract}
"En los últimos 25 años, la escritura de las mujeres invade redacciones de libros y revistas, de diarios y noticiarios; de redacciones de revistas electrónicas y páginas Web, somos hasta el 50 por ciento de las cabezas que elaboran los contenidos de programas de radio y televisión, nuestras voces e imágenes comparten la pantalla televisiva en menor escala, pero en forma creciente" (LOVERA, 2007, p. 22).
\end{abstract}

Un camino hacia la igualdad de género en los medios de comunicación en México, impulsó la estrategia de la creación de Comunicación e Información de la Mujer [8] (CIMAC), que ha generado una red de intercambio y comunicación importante, a través de estrategias planificadas, que permitan fomentar la organización de las trabajadoras de la escritura y el manejo de los medios; periodistas, escritoras, publicistas e intelectuales en el país (LOVERA, 2007).

Por otro lado, el multiculturalismo, la transversalidad y la globalización, ha impactado y modificado la manera de entender, interpretar y ejercer nuestros derechos humanos, es decir, ahora, en pleno siglo XXI, se habla de cuatro "olas" o "generaciones" de derechos humanos, no obstante, el eje central que prevalece en estas "generaciones" es la comunicación, independientemente de su formato.

El tema no ha concluido, falta mucho para que esto suceda, máxime si los procesos de comunicación siguen avanzando, por tal, necesitamos reconocer, que la sociedad continúa hacia el conocimiento de los derechos humanos y debe progresar todavía hacia las diversas perspectivas de género, las cuales antes se identificaban como hombre y mujer, hoy, eso se ya no encaja con la realidad. Es por eso que la UNESCO, - a favor de la igualdad para todos-, publica en 2016, un informe denominado "Out In the Open. Education sector responses to violence based on sexual orientation and gender identity/expression", donde justifica que los 
estudiantes que no son percibidos de acuerdo a las normas sexuales de género vigentes, incluidas lesbianas, gays, bisexuales y personas transexuales, identificados como LGTB, están más predispuestos a ser acosados y a ser víctimas de la violencia, llamada homofóbica y transfóbica. Lo que significa, que si aún no hemos aprendido a establecer protocolos justos e igualitarios en todos los niveles con las mujeres, todavía debemos trabajar a favor que los grupos identificados como LGTB, quienes son tan importantes y valiosos como lo podría ser cualquier ser humano.

Por consiguiente, debemos preguntarnos, cuál es el papel de la investigación en comunicación en sociedades que no conciben una visión de igualdad entre hombres, mujeres y LGTB.

Cuando nos percatemos de esto, hay reconocer entonces, que los medios de información son una institución cultural concluyente, que dada su centralidad, influyen en los pensamientos al establecer los estereotipos de género y las reglas de conducta sexual a través de los relatos informativos y las representaciones ficticias de lo social (CABALLERO, 2015, p.13).

\section{Notas}

[1] Disponível em: <http://www.unwomen.org/es >. Acesso em: 20 dez. 2017.

[2] Disponível em: <http://www.unesco.org/new/es/communication-andinformation/intergovernmental-programmes/ipdc/about-ipdc/>. Acesso em: 20 dez. 2017.

[3] Disponível em: $<$ http://unesdoc.unesco.org/images/0023/002310/231069s.pdf $>$. Acesso em: 20 dez. 2017.

[4] Disponível em: <https://www.cpj.org/es/>. Acesso em: 20 dez. 2017.

[5] Somalia, Siria, Irak, Sudán del Sur, Filipinas, México, Pakistán, Brasil, Rusia, Blangadesh, Nigeria e India.

[6] 37 periodistas han sido asesidados en México desde 1992, donde el motivo de su muerte ha sido confirmado por el CPJ, es decir, se ha investigado y comprobado, que el periodista fue asesinado en represalia directa por su trabajo (Comité para la Protección de los Periodistas, 2016, s/p).

[7] Disponível em: <http://www.unesco.org/new/en/communication-andinformation/resources/publications-and-communication-materials/publications/fulllist/model-curricula-for-journalism-education-a-compendium-of-new-syllabi/>. Acesso em: 20 dez. 2017. 
[8] CIMAC, fundada en 1988. Disponível em: < http://www.cimac.org.mx/>. Acesso em: 21 dez. 2017.

\section{Referências}

ANIMAL POLÍTICO. 2016 bate récord de periodistas asesinados durante gobierno de Peña: van 10 en 9 meses. 2016. Disponível em:

<http://www.animalpolitico.com/2016/11/2016-asesinatos-periodistas-pena/>. Acesso em: 21 dez. 2017.

BOAFO, Kwame. Comunicación y derechos humanos: el papel de la Unesco, en Montiel, A. (Coord.). Comunicación y derechos humanos. Universidad Nacional Autónoma de México, Centro de Investigaciones Interdisciplinarias en Ciencias y Humanidades, Asociación Internacional de Estudios en Comunicación Social, 2012. Disponível em: <http://computo.ceiich.unam.mx/webceiich/docs/libro/Comunicacion y derechos humanos.pdf>. Acesso em: 21 dez. 2017.

BURCH, Sally. Género y comunicación: la agenda de las mujeres en comunicación para el nuevo siglo. En Agencia Latinoamérica de Información ALAI. 2007.

BUSTAMANTE, Javier. Hacia la cuarta generación de Derechos Humanos: repensando la condición humana en la sociedad tecnológica. In Revista iberoamericana de Ciencia, Tecnología, Sociedad e innovación, v.1, n.3. 2001. Disponível em: $<$ https://dialnet.unirioja.es/servlet/articulo?codigo=741542>. Acesso em: 21 dez. 2017.

BRUNO, Daniela P., y GUERRINI, Lucía. Cuadernillo I. Colección' Comunicación, desarrollo y derechos humanos'. Proyecto de Capacitación UNICEF-EDUPAS Comunicación Educativa. 2006. Disponível em: <https://www.unicef.org/argentina/spanish/EDUPAScuadernillo-1(1).pdf $>$. Acesso em: 21 dez. 2017.

CABALLERO, Francisco Sierra. Comunicación y Género. Agendas y cultura de investigación. Chasqui. Editorial. In Revista Latinoamericana de Comunicación, n. 135, p. 09-14. 2017. Disponível em:

<http://www.revistachasqui.org/index.php/chasqui/article/view/3340>. Acesso em: Acesso em: 21 dez. 2017.

CALLAMARD, Agnes. Comunicación y derechos humanos: Artículo 19, en Montiel, Aimée (Coord.). Comunicación y derechos humanos. Universidad Nacional Autónoma de México, Centro de Investigaciones Interdisciplinarias en Ciencias y Humanidades, Asociación Internacional de Estudios en Comunicación Social. 2012. Disponível em: 
$<$ http://computo.ceiich.unam.mx/webceiich/docs/libro/Comunicacion_y derechos humanos.pdf >. Acesso em: 21 dez. 2017.

DELORS, Jacques, AMAGI Isao, CARNEIRO Roberto, CHUNG Fay, GEREMEK Bronislaw, GORHAM William, KORNHAUSER Aleksandra et al. La Educación encierra un Tesoro: Informe a la UNESCO de la Comisión Internacional sobre la Educación para el Siglo XXI. Compendio. Santillana Ediciones UNESCO. 1997. $<$ http://unesdoc.Unesco.org/images/0010/001095/109590so.pdf > . Acesso em: 21 dez. 2017.

FAUR, Eleonor. ¿Escrito en el cuerpo? Género y derechos humanos en la adolescencia. En Checa Susana, comp. Género, sexualidad y derechos reproductivos en la adolescencia. Buenos Aires: Paidós, 2003.

PHILIPP, Rita. Derechos humanos y género. Cad. Cedes, v.30, n.81, p.135-155. 2010. Disponível em: <http://www.scielo.br/pdf/ccedes/v30n81/a02v3081>. Acesso em: 21 dez. 2017.

FRAU-MEIGS, Divina. Educación en medios y derechos humanos, en Montiel, Aimée (Coord.). Comunicación y derechos humanos. Universidad Nacional Autónoma de México, Centro de Investigaciones Interdisciplinarias en Ciencias y Humanidades, Asociación Internacional de Estudios en Comunicación Social. 2012.

GALLAGHER, Margaret. Los derechos humanos y a comunicar de las mujeres, en Montiel, A. (Coord.). Comunicación y derechos humanos. Universidad Nacional Autónoma de México, Centro de Investigaciones Interdisciplinarias en Ciencias y Humanidades, Asociación Internacional de Estudios en Comunicación Social. 2012.

García-Ruiz, R., Aguaded Gómez, J. I., \& Rodríguez Vázquez, A. I. Propuesta de alfabetización mediática ante los estereotipos de género en los medios de comunicación. Resultados de la valoración de" Rostros de Mujer". Prisma Social, v.13, p. 576-609, 2014. Disponível em:

<http://www.redalyc.org/html/35.37/353744532016/>. Acesso em: 21 dez. 2017.

LEWY, Frank La Rue. Comunicación y derechos humanos: La relatoría especial de las Naciones Unidas, en Montiel, A. (Coord.). Comunicación y derechos humanos. Universidad Nacional Autónoma de México, Centro de Investigaciones Interdisciplinarias en Ciencias y Humanidades, Asociación Internacional de Estudios en Comunicación Social. p.55-62. 2012.

LOVERA, Sara. Comunicación y género. Comunicación e cidadanía. In Revista internacional de xornalismo social = social journalism international review, v.1, p. 19-24. 2007. Disponível em:

$<$ https://dialnet.unirioja.es/descarga/articulo/2399814.pdf > . Acesso em: 21 dez. 2017. 
MONTIEL, Aimée. Comunicación y derechos humanos. Universidad Nacional Autónoma de México, Centro de Investigaciones Interdisciplinarias en Ciencias y Humanidades, Asociación Internacional de Estudios en Comunicación Social. 2012.

NACIONES UNIDAS. Declaración Universal de Derechos Humanos, 2015.

Disponível em :

<http://www.un.org/es/documents/udhr/UDHR_booklet_SP_web.pdf $>$. Acesso em: 21 dez. 2017.

KI-MOON, Ban. Prologo. Declaración Universal de Derechos Humanos. 2015. Disponível em: <http://www.un.org/es/documents/udhr/UDHR booklet SP web.pdf >. Acesso em: 21 dez. 2017.

ONU MUJERES, 2016. Disponível em: <http://www.unwomen.org/es > . Acesso em: 21 dez. 2017.

PASQUALI, Antonio. Derechos del hombre y comunicación en América Latina, en Montiel, A. (Coord.). Comunicación y derechos humanos. Universidad Nacional Autónoma de México, Centro de Investigaciones Interdisciplinarias en Ciencias y Humanidades, Asociación Internacional de Estudios en Comunicación Social. 2012.

SENDÍN GUTIÉRREZ, Juan Carlos. Unesco y la mejora de la formación de los periodistas. Un compendio de propuestas. In Index. comunicación: Revista científica en el ámbito de la Comunicación Aplicada, v.5, n.2, p. 9-20. 2015. Disponível em: $<$ https://dialnet.unirioja.es/servlet/articulo? codigo=5404903>. Acesso em: $21 \mathrm{dez}$. 2017.

TALAVERA, Pedro. Diálogo intercultural y universalidad de los derechos humanos. Revista IUS, v. 5, n. 28, p. 7-38. 2011. Disponível em: <http://www.scielo.org.mx/pdf/rius/v5n28/v5n28a2.pdf>. Acesso em: 21 dez. 2017.

SREBERNY, Annabelle. PREFACIO en Montiel, A. (Coord.). Comunicación y derechos humanos. Universidad Nacional Autónoma de México, Centro de Investigaciones Interdisciplinarias en Ciencias y Humanidades, Asociación Internacional de Estudios en Comunicación Social. 2012.

United Nations International Children's Emergency Fund (UNICEF). Female Genital Mutilation/Cutting: A Global Concern, 2016. Disponível em:

$<$ https://www.unicef.org/media/files/FGMC 2016 brochure final UNICEF SPRE AD.pdf $>$. Acesso em: 21 dez. 2017.

United Nations Educational, Scientific and Cultural Organization (UNESCO). Indicadores de Género para Medios de Comunicación. Marco de indicadores para evaluar la sensibilidad en materia de género en las operaciones y contenidos mediáticos, 2014. Disponível em:< 
http://unesdoc.Unesco.org/images/0023/002310/231069s.pdf > . Acesso em: $21 \mathrm{dez}$. 2017.

United Nations Educational, Scientific and Cultural Organization (UNESCO). Communication and Information. Model curricula for journalism education: a compendium of new syllabi, 2014.

http://www.Unesco.org/new/en/communication-andinformation/resources/publications-and-communicationmaterials/publications/full-list/model-curricula-for-journalism-education-acompendium-of-new-syllabi/

United Nations Educational, Scientific and Cultural Organization (UNESCO) Comunicación e Información. Género y medios de comunicación: lograr el equilibrio adecuado. 2016.

Disponível em: <http://www.Unesco.org/new/es/communication-andinformation/intergovernmental-programmes/ipdc/about-ipdc/intergovernmentalcouncil/28th-session-of-ipdc-council/gender-and-media-getting-the-balanceright/>. Acesso em: 21 dez. 2017.

United Nations Educational, Scientific and Cultural Organization (UNESCO) Out In the Open. Education sector responses to violence based on sexual orientation and gender identity/expression. 2016. Disponível em: < http://unesdoc.Unesco.org/images/0024/002447/244756e.pdf>. Acesso em: $21 \mathrm{dez}$. 2017. 